\title{
Erratum: Coherent control of collective atom phase for ultralong, inversion-free photon echoes [Phys. Rev. A 85, 031402(R) (2012)]
}

\author{
Byoung S. Ham* \\ (Received 6 October 2016; published 31 October 2016)
}

DOI: 10.1103/PhysRevA.94.049905

Equations (1) and (2) are corrected for the silent echo $E 1$ in Fig. 1(a), where $E 1$ does not affect final photon echo $E 2$ that is under no population inversion. For this, $\mathbf{k}_{D}=-\mathbf{k}_{R R}$ and $\mathbf{k}_{W}=\mathbf{k}_{R}=\mathbf{k}_{R R}$, where $\mathbf{k}_{i}$ is a propagation wave vector of each optical pulse $i$. Then the phase matching conditions for $E 1$ and $E 2$ are, respectively,

$$
\begin{gathered}
\mathbf{k}_{E 1}=\mathbf{k}_{R}+\mathbf{k}_{W}-\mathbf{k}_{D}=-3 \mathbf{k}_{D}, \\
\mathbf{k}_{E 2}=2 \mathbf{k}_{R R}-\mathbf{k}_{E 1}=2 \mathbf{k}_{R R}-\left(\mathbf{k}_{R}+\mathbf{k}_{W}\right)+\mathbf{k}_{D}=\mathbf{k}_{D} .
\end{gathered}
$$

Unlike the spatial phase mismatch between $E 1\left(\mathbf{k}_{E 1}=-3 \mathbf{k}_{D}\right)$ and $D\left(\mathbf{k}_{D}\right)$ along the medium length $L(L \gg \lambda)$ due to $\left|k_{E 1}-k_{D}\right| L=\frac{2 \pi}{\lambda}|n(3 \omega)-n(\omega)| L \gg \pi$ in Eq. (1) resulting in a silent echo [1], echo $E 2$ stays in phase due to $\left|k_{E 2}-k_{D}\right| L=0$ in Eq. (2).

I thank A. Faraon, J. Bartholomew, T. Zhong, and J. Kindem at Caltech for useful discussions regarding double rephasing in gradient echoes. This work was supported by ICT R\&D program of MSIP/IITP (1711028311: Reliable crypto-system standards and core technology development for secure quantum key distribution network) and GIST-Caltech Program in 2016.

[1] V. Damon, M. Bonarota, A. Louchet-Chauvet, T. Chaneliere, and J.-L. Le Gouet, New J. Phys. 13, 093031 (2011). 